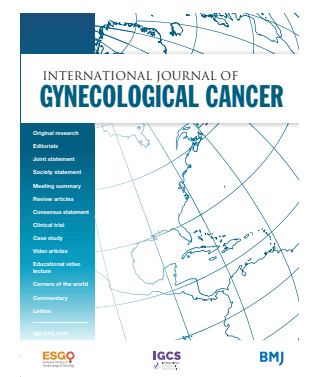

${ }^{1}$ Department of Surgical Oncology, Institut Claudius Regaud - Institut Universitaire du Cancer de Toulouse Oncopole, Toulouse, France ${ }^{2}$ Team 1, Tumor Immunology and Immunotherapy, INSERM, CRCT, Toulouse, France ${ }^{3}$ Team 19, ONCOSARC Oncogenesis of sarcomas, INSERM CRCT, Toulouse, France

Correspondence to Dr Alejandra Martinez, Department of Surgical Oncology, Institut Claudius Regaud - Institut Universitaire du Cancer de Toulouse Oncopole, 1 Avenue Irène Joliot-Curie 31059 Toulouse Cedex 9, Toulouse, France; martinez.alejandra@iuctoncopole.fr

Accepted 14 August 2020 Published Online First 21 September 2020

\section{Transdiaphragmatic and transxiphoid cardiophrenic lymph node resection step-by- step in advanced ovarian cancer}

Carlos Martínez-Gómez (D) , 1,2 Martina Aida Angeles, ${ }^{1}$ Hélène Leray, ${ }^{1}$ Yann Tanguy Le Gac, ${ }^{1}$ Gwenael Ferron, ${ }^{1,3}$ Alejandra Martinez (D) 1,2

\section{ABSTRACT}

Upper abdominal disease is present in the majority of patients with advanced-stage epithelial ovarian, fallopian tube, and primary peritoneal cancer. Extensive upper abdominal procedures have been associated with higher rates of complete cytoreduction and improved survival. ${ }^{1}$ Cardiophrenic lymph nodes (CPLNs) can be involved in patients with extensive upper abdominal tumor burden, as they receive the lymphatic drainage from the diaphragm and the abdominal cavity. ${ }^{2}$ Surgical removal of enlarged CPLNs helps to identify stage IV disease and to decrease the rate of residual disease. CPLN involvement should not preclude primary cytoreductive surgery when complete abdominal cytoreduction can be achieved. ${ }^{3}$ CPLN resection is associated with low complication rates, including pleural effusion, pneumothorax, and pneumonia. Videoassisted thoracic surgery and transdiaphragmatic approach represent two alternative approaches for their resection. ${ }^{4}$

Video 1 shows step-by-step the surgical technique for removing enlarged lymph nodes located in the lowest part of the anterior, medium, and posterior mediastinum by a laparotomic approach in three patients who underwent a primary cytoreductive surgery for high-grade serous ovarian cancer. Transxiphoid approach is employed to remove retroxiphoid lymph nodes, which are found in the anterior mediastinum. The xiphoid process can optionally be resected and the ventral diaphragmatic fibers incised to give access to the anterior mediastinum. This virtual space is developed, and enlarged nodes are identified and removed without pleural opening. The tendinous diaphragmatic opening can be closed with interrupted stitches.
Still figure. Schematic picture with the two different approaches for mediastinal lymph node resection (abdominal view). (Abbreviations: $\mathrm{LN}$, lymph node)

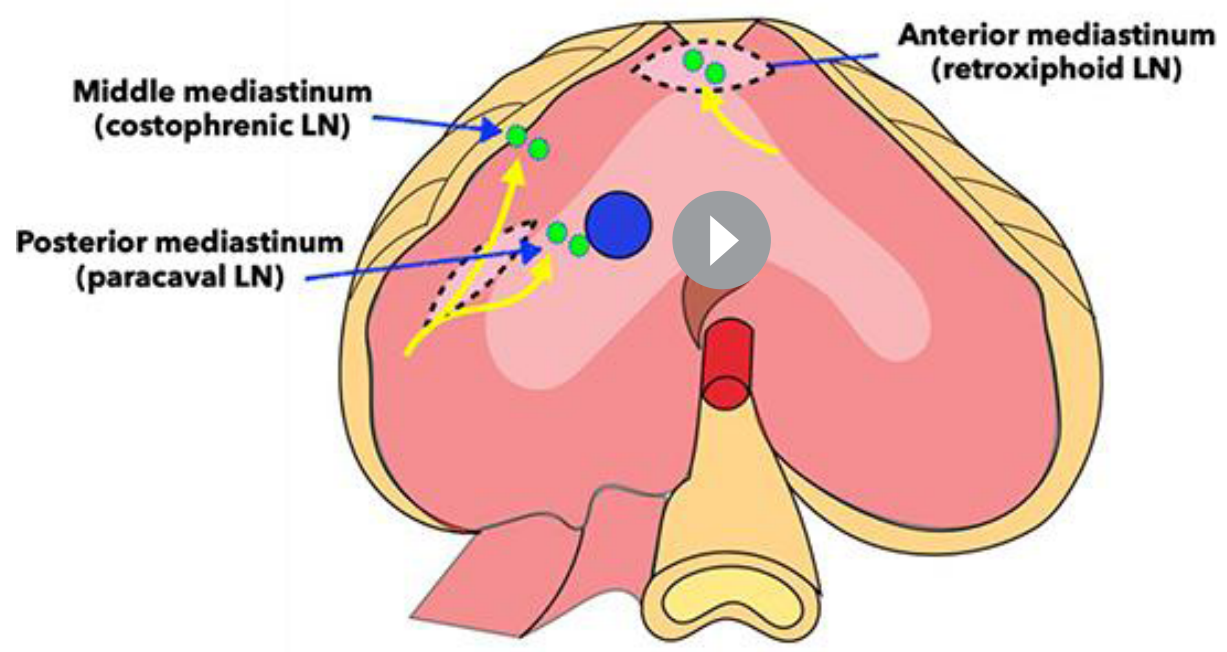

To cite: Martínez-Gome
Angeles MA, Leray $\mathrm{H}$ et al. Int J Gynecol Cancer 2020;30:1646-1647.

Video 1. Transdiaphragmatic and transxiphoid cardiophrenic lymph node resection. 
Right transdiaphragmatic approach is useful for removing enlarged right costophrenic and paracaval lymph nodes, situated in the medium and posterior mediastinum, respectively. This technique requires a previous hepatic mobilization before the opening of the right diaphragm and the parietal pleura. The fat pads of the medium and posterior mediastinum are examined to remove enlarged lymph nodes. The diaphragm is closed with a running suture and the pneumothorax can be evacuated employing a chest tube. It is possible to perform an air leak test to verify diaphragmatic tightness.

In conclusion, transxiphoid approach is useful for resecting retroxiphoid lymph nodes while transdiaphragmatic approach can be used for costophrenic and paracaval lymph node resection.

Twitter Carlos Martínez-Gómez @cmartgomez, Martina Aida Angeles @AngelesFite and Alejandra Martinez @Alejandra

Acknowledgements MAA acknowledges grant support from "la Caixa" Foundation, Barcelona, Spain (ID 100010434). Fellowship code LCF/BQ/ EU18/11650038).

Contributors $\mathrm{CM}-\mathrm{G}$ : conceptualization, video editing, writing - original draft. MAA: conceptualization, surgery and video editing, writing - original draft. $\mathrm{HL}$ : conceptualization, project administration, surgery. YTLG: conceptualization, project administration, surgery. GF: conceptualization, project administration, supervision, writing - review. AM: conceptualization, project administration, surgery and video recording, supervision, writing - review.
Funding The authors have not declared a specific grant for this research from any funding agency in the public, commercial or not-for-profit sectors.

Competing interests GF reports personal fees from Olympus outside of the submitted work.

Patient consent for publication Not required.

Provenance and peer review Not commissioned; externally peer reviewed.

Data availability statement All data relevant to the study are included in the article.

ORCID iDs

Carlos Martínez-Gómez http://orcid.org/0000-0002-9652-7880

Alejandra Martinez http://orcid.org/0000-0002-7633-3536

\section{REFERENCES}

1 Zivanovic O, Eisenhauer EL, Zhou Q, et al. The impact of bulky upper abdominal disease cephalad to the greater omentum on surgical outcome for stage IIIC epithelial ovarian, fallopian tube, and primary peritoneal cancer. Gynecol Oncol 2008;108:287-92.

$2 \mathrm{Kim} \mathrm{T-H,} \mathrm{Lim} \mathrm{MC,} \mathrm{Kim} \mathrm{SI,} \mathrm{et} \mathrm{al.} \mathrm{Preoperative} \mathrm{prediction} \mathrm{of}$ cardiophrenic lymph node metastasis in advanced ovarian cancer using computed tomography. Ann Surg Oncol 2016;23:1302-8.

3 Cowan RA, Tseng J, Murthy V, et al. Feasibility, safety and clinical outcomes of cardiophrenic lymph node resection in advanced ovarian cancer. Gynecol Oncol 2017;147:262-6.

4 Lim MC, Lee H-S, Jung DC, et al. Pathological diagnosis and cytoreduction of cardiophrenic lymph node and pleural metastasis in ovarian cancer patients using video-assisted thoracic surgery. Ann Surg Oncol 2009;16:1990-6. 